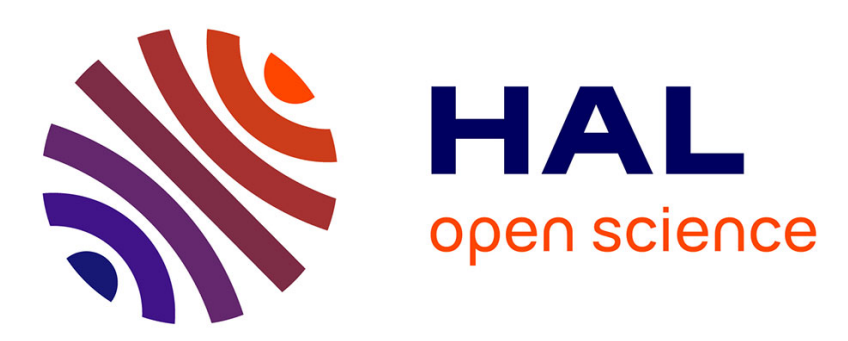

\title{
Mesure simultanée du pouvoir thermoélectrique et de la diffusivité thermique par la méthode de l'impulsion thermique
}

\author{
A. Siblini, E. Souquet, G. Mesnard
}

\section{- To cite this version:}

A. Siblini, E. Souquet, G. Mesnard. Mesure simultanée du pouvoir thermoélectrique et de la diffusivité thermique par la méthode de l'impulsion thermique. Revue de Physique Appliquée, 1982, 17 (1), pp.29-35. 10.1051/rphysap:0198200170102900 . jpa-00244966

\section{HAL Id: jpa-00244966 https://hal.science/jpa-00244966}

Submitted on 1 Jan 1982

HAL is a multi-disciplinary open access archive for the deposit and dissemination of scientific research documents, whether they are published or not. The documents may come from teaching and research institutions in France or abroad, or from public or private research centers.
L'archive ouverte pluridisciplinaire HAL, est destinée au dépôt et à la diffusion de documents scientifiques de niveau recherche, publiés ou non, émanant des établissements d'enseignement et de recherche français ou étrangers, des laboratoires publics ou privés. 


\title{
Mesure simultanée du pouvoir thermoélectrique et de la diffusivité thermique par la méthode de l'impulsion thermique
}

\author{
A. Siblini (*), E. Souquet et G. Mesnard \\ Université Claude-Bernard de Lyon, Laboratoire de Physique Electronique, 69622 Villeurbanne Cedex, France
}

(Reçu le 23 juillet 1981, révisé le 28 septembre 1981, accepté le 7 octobre 1981)

\begin{abstract}
Résumé. - La méthode de l'impulsion thermique, obtenue par flash optique ou par laser, pour la mesure des caractéristiques thermiques des corps (diffusivité, conductibilité, chaleur spécifique) est bien connue. Nous avons étendu la méthode à la mesure simultanée du pouvoir thermoélectrique. Connaissant l'énergie absorbée par la face antérieure de l'échantillon, l'évolution en température de la face opposée et la f.é.m. résultante obtenue entre les deux faces, on détermine le pouvoir thermoélectrique sans passer par la mesure du gradient de température à travers l'échantillon.

Abstract. - The thermal pulse method, using an optical flash or a laser, for measuring the thermal characteristics of materials (diffusivity, conductivity, specific heat) is well known. We have extended the method to the simultaneous measurement of thermoelectric power. Knowing the energy absorbed by the front surface of the sample, the variation of the temperature of the opposite surface and the resultant emf obtained between the two faces, one determines the thermoelectric power without necessity of measurement of the temperature gradient across the sample.
\end{abstract}

1. Généralités sur la mesure du pouvoir thermoélectrique. - On peut distinguer deux méthodes générales pour la mesure du pouvoir thermoélectrique (P.T.E.), la méthode intégrale et la méthode différentielle. Cette dernière est la plus commode pour les échantillons de faibles dimensions. Elle consiste à appliquer une faible différence $\Delta T$ de température entre les deux faces, supposées planes et parallèles, de l'échantillon et à mesurer la différence de potentiel $\Delta E$ qui en résulte. Le P.T.E. est

$$
Q_{\mathrm{v}}=-\frac{\Delta E}{\Delta T} \text {. }
$$

Des méthodes statiques ou dynamiques sont employées. Les méthodes statiques entraînent de nombreuses difficultés et imprecisions :

- maintien d'un gradient stable pendant un temps assez long,

- existence de f.é.m. parasites,

- erreurs dues aux pertes thermiques, etc...

De nombreux auteurs ont montré l'intérêt des mesures en régime dynamique. Parmi les solutions proposées, citons celle de Rowe et Schröder [1] qui consiste à appliquer à l'échantillon des échelons de

(*) Nouvelle adresse : Université Libanaise, Faculté des Sciences, Hadeth, Liban.

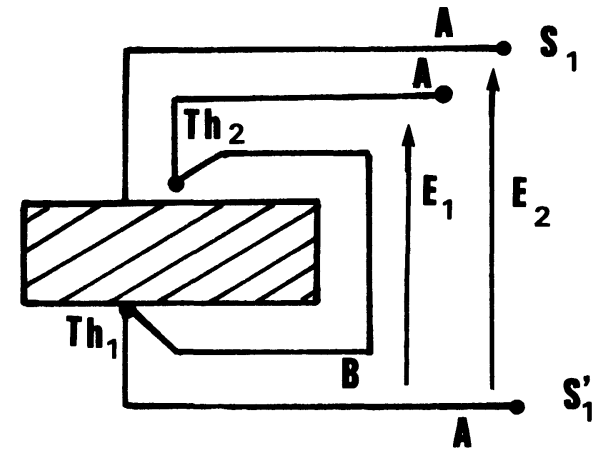

Fig. 1. - Schéma de la méthode de Caskey et al. pour les mesures de pouvoir thermoélectrique.

[Diagram of Caskey et al.'s method for thermoelectric power measurements.]

température $\Delta T_{n}$ successifs et à mesurer les $\Delta E_{n}$ correspondants. Un procédé plus rapide [2] utilise le schéma de montage de la figure 1 fonctionnant en régime variable quelconque. Les deux thermocouples $\mathrm{Th}_{1}$ et $\mathrm{Th}_{2}$ montés en différentiel permettent la mesure de $\Delta T$ par l'intermédiaire de la f.é.m. $E_{1}$ tandis que $\Delta E$ n'est autre que la tension $E_{2}$ mesurée simultanément entre $S_{1}$ et $S_{1}^{\prime}$. Le thermocouple $T h_{1}$ ne peut être en contact direct avec l'échantillon, sa résistance électrique étant alors en parallèle avec celle de l'échan- 


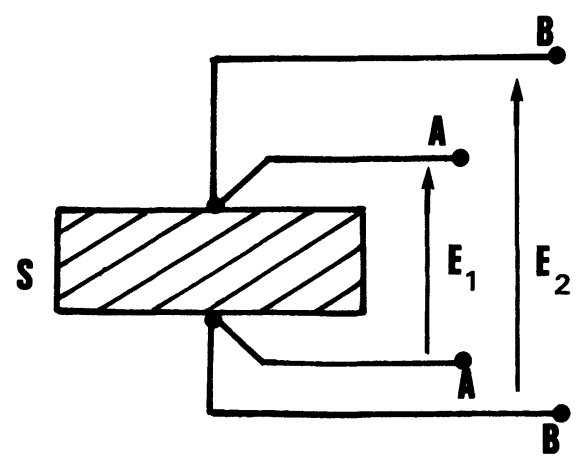

Fig. 2. - Schéma de la méthode de Conan et al. pour les mesures de pouvoir thermoélectrique.

[Diagram of Conan et al.'s method of thermoelectric power measurements.]

tillon; l'erreur dans la mesure de $\Delta T$ peut donc être considérable.

Conan et al. [3] évitent cet inconvénient avec le schéma de la figure 2. $E_{1}$ et $E_{2}$ représentent ici respectivement les f.é.m. de Seebeck du matériau $S$ constituant l'échantillon par rapport aux métaux différents $A$ et $B$ utilisés pour réaliser les deux thermocouples. Leur différence $E$ est la f.é.m. de Seebeck entre $A$ et $B$ pour la même différence de température. La mesure simultanée de $E_{1}$ et $E_{2}$ permet donc d'accéder au P.T.E. $Q_{\mathrm{S} / \mathrm{A}}$ de $\mathrm{S}$ par rapport à $\mathrm{A}$, connaissant le pouvoir thermoélectrique $Q_{\mathrm{B} / \mathrm{A}}$ de $\mathrm{B}$ par rapport à $\mathrm{A}$, à la même température, par la relation

$$
Q_{\mathrm{S} / \mathbf{A}}=\frac{E_{1}}{E} Q_{\mathbf{B} / \mathbf{A}}
$$

sans qu'il y ait à déterminer $\Delta T$. Mais une bonne précision exige que $E_{1}, E_{2}$ et $E$ soient du même ordre de grandeur.

Ces auteurs ont montré la validité des mesures en régime dynamique et l'intérêt qu'il y a à éviter la mesure directe de $\Delta T$. Ces deux remarques nous ont conduit à développer la méthode qui suit. Nous avons utilisé la méthode de mesure de la diffusivité thermique par flash pour mesurer aussi le P.T.E. ; les équations de la propagation de la chaleur permettent alors de connaître le gradient thermique à travers l'échantillon, sans le mesurer; simultanément nous déterminons, par un procédé classique, les grandeurs thermiques (diffusivité thermique, conductibilité thermique, chaleur spécifique). Et nous bénéficions des avantages d'une mesure en régime transitoire :

- rapidité de la mesure,

- suppression des problèmes inhérents aux mesures en continu, tels que la stabilité à long terme,

- disparition des problèmes d'obtention de bons contacts thermiques.

2. Théorie de la méthode utilisée. -2.1 MESURES THERMIQUES. - Parker, Jenkins, Butler et Abbott [4]

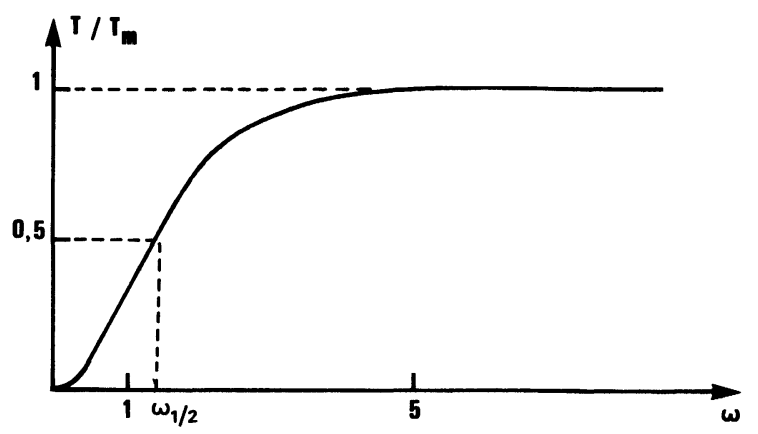

Fig. 3. - Variation de la température après l'impulsion thermique.

[Temperature variation after the thermal pulse.]

ont développé la théorie de la méthode du flash pour la mesure de la diffusivité thermique. Nous allons la rappeler brièvement. L'énergie d'une impulsion de lumière très brève est absorbée par la surface $A$ d'un échantillon plat. L'évolution de la température de la face opposée B est enregistrée à l'aide d'un thermocouple. La figure 3 donne la forme de la courbe obtenue, dont nous allons donner la théorie. $T$ représente ici l'élévation de température, $T_{\mathrm{m}}$ sa valeur finale; $\omega$ est proportionnel au temps $t$.

Soit $L$ l'épaisseur de l'échantillon et $D$ sa masse volumique, la chaleur spécifique du corps est

$$
C=\frac{Q}{L T_{\mathrm{m}} D}
$$

$Q$ étant la quantité d'énergie reçue par unité de surface.

En posant

$$
V=T / T_{\mathrm{m}}
$$

on trouve d'autre part

$$
V=1+2 \sum_{n=1}^{\infty}(-1)^{n} \exp \left(-n^{2} \omega\right)
$$

avec

$$
\omega=\pi^{2} \alpha t / L^{2}
$$

où $\alpha$ est la diffusivité thermique du corps. Pour la valeur particulière $V=0,5$, l'équation (2) donne la valeur particulière

$$
\omega_{1 / 2}=1,38
$$

qui correspond à un temps $t_{1 / 2}$.

De l'équation (3) on peut alors tirer $\alpha$, soit :

$$
\alpha=\frac{1,38 L^{2}}{\pi^{2} t_{1 / 2}} .
$$

Enfin la conductibilité thermique $K$ est donnée par la formule

$$
K=\alpha C D
$$


2.2 Mesure DU POUVOIR THERMOÉLECTRIQUE. On détermine la f.é.m. $\Delta E=f(t)$ qui apparait entre les deux faces de l'échantillon. On a :

$$
Q_{\mathrm{v}}=-\frac{\Delta E}{\Delta T}
$$

L'équation (2) nous permettait de calculer à tout instant l'élévation de la température de la face $B$ de l'échantillon. Pour la face A on trouve, en introduisant la quantité $V_{\mathrm{A}}$ analogue à $V$,

$$
V_{\mathrm{A}}=1+2 \sum_{n=1}^{\infty} \exp \left(-n^{2} \omega\right) \text {. }
$$

Par différence on trouve pour la différence $\Delta T$ de température entre $\mathbf{A}$ et $\mathbf{B}$ :

$$
\Delta T=4 T_{\mathrm{m}} \sum_{\substack{1 \\ n^{2} \text { impair }}}^{\infty} \exp \left(-n^{2} \omega\right) .
$$

Il convient de faire la mesure lorsque $\Delta T$ est devenu assez faible. Or la somme précédente converge rapidement à partir de $\omega=0,75$; avec une erreur inférieure à $0,25 \%$, on peut prendre alors

$$
\Delta T=4 T_{\mathrm{m}} \exp (-\omega) .
$$

Finalement

$$
Q_{\mathrm{v}}=\frac{-\Delta E}{4 T_{\mathrm{m}} \exp (-\omega)}
$$

ou

$$
\Delta E=-4 Q_{\mathrm{v}} T_{\mathrm{m}} \exp (-\omega) .
$$

On a alors plusieurs méthodes pour obtenir $Q_{v}$ :

a) $\mathrm{Si}$ on a préalablement déterminé $\alpha$, il suffit d'appliquer la formule (9) à un instant donné pour lequel on lit $\Delta E$ et on calcule $\omega$.

b) Il vaut mieux tirer parti de toute l'évolution pour trouver $Q_{v}$ et en même temps $\alpha$. On trace la courbe de $\log |\Delta E|=f(t)$. D'après l'équation (10) ce doit être une droite. Sa pente est égale à $-\pi^{2} \alpha / L^{2}$ et son ordonnée à l'origine est $\log \left(4\left|Q_{\mathrm{v}}\right| T_{\mathrm{m}}\right)$.

c) On peut aussi éliminer $\omega$ entre (2) et (9) de façon à relier simplement $\Delta E$ à $T$ au cours du temps. Quand on peut ne retenir que le premier terme du développement de $V$ dans (2), c'est-à-dire au bout d'un temps assez long, on trouve

$$
Q_{\mathrm{v}}=-\frac{\Delta E}{2\left(T_{\mathrm{m}}-T\right)}
$$

3. Conditions de validité de la méthode. - La théorie exposée ci-dessus suppose :

a) une impulsion de durée infiniment courte, avec une onde plane,

b) une absorption uniforme de l'échantillon, supposé isotrope,

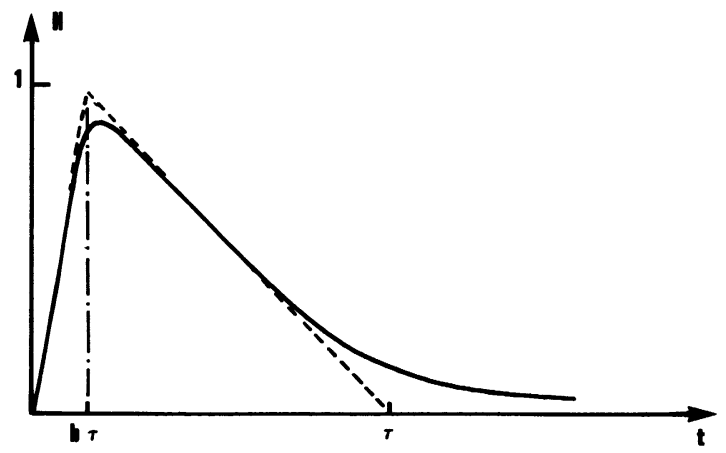

Fig. 4. - Variation de la puissance relative émise par la lampe flash au cours du temps.

[Relative variation of the power emitted by the flash lamp vs. temperature.]

c) des pertes thermiques négligeables.

Les conditions $b$ ) et $c$ ) sont technologiquement réalisables; nous le verrons en exposant les procédés expérimentaux utilisés.

Il est par contre souvent plus délicat de satisfaire de façon satisfaisante la condition a). Heckmann [5] a étudié l'influence de la durée finie d'impulsion sur la précision de la mesure de $\alpha$. La figure 4 montre, en valeur relative $h$, l'évolution temporelle de la puissance de l'impulsion thermique et l'approximation triangulaire utilisée. $\tau$ est la durée effective de l'impulsion, $b \tau$ est le temps de montée. Soit alors $\alpha$ la diffusivité réelle et $\alpha_{0}$ la diffusivité expérimentale obtenue à partir de $t_{1 / 2}$. Nous donnons pour deux valeurs de $b$, sur le graphique de la figure 5 , la variation de $\alpha / \alpha_{0}$ en fonction de $\tau / t_{1 / 2}$. Il apparaît que des résultats à mieux que $3 \%$ environ ne peuvent être obtenus que pour $\tau<t^{1 / 2} / 10$. Suivant la source d'énergie utilisée (flash ou laser), on se placera dans ces conditions en choisissant une épaisseur $L$ suffisante.

Il faut noter que, si l'on s'intéresse au comportement observé après un temps supérieur à $t_{1 / 2}$, l'erreur devient plus faible; or c'est le cas dans la méthode de mesure simultanée de $\alpha$ et $Q_{v}$ indiquée plus haut. Il

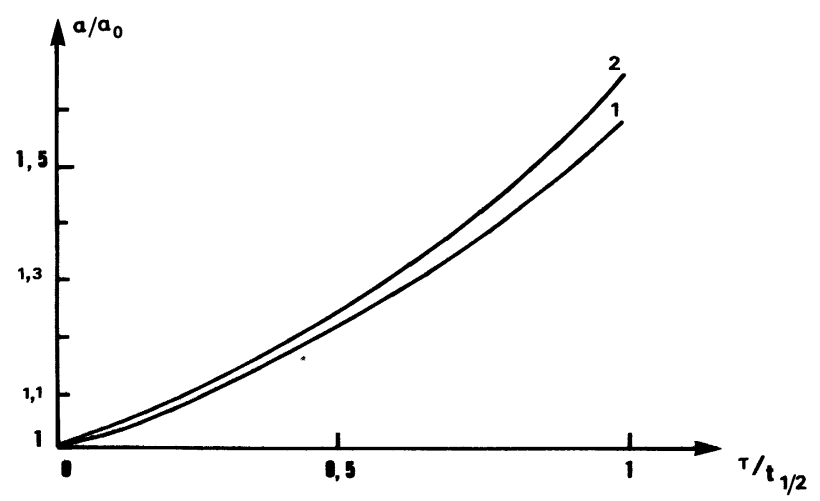

Fig. 5. - Corrections de diffusivité : la courbe 1 correspond à $b=0$, la courbe 2 à $b=0,1$.

[Corrections for the diffusivity : curve 1 corresponds to $b=0$, curve 2 to $b=0.1$.] 
est d'ailleurs de toute façon souhaitable d'opérer dans des conditions où le gradient thermique n'est pas trop élevé afin d'éviter les non-linéarités.

Il faut d'autre part tenir compte de l'échauffement sur les propriétés du corps ; la face avant peut atteindre des températures élevées, l'énergie étant délivrée en un temps très bref. Il est nécessaire de connaître la température maximale $T_{\mathrm{f}}$ atteinte pour $Q$ donné, afin de régler $Q$ pour éviter toute détérioration ou modification structurale de l'échantillon.

Pour trouver $T_{\mathrm{f}}$, introduisons à nouveau une approximation triangulaire de l'onde thermique incidente (Fig. 4) et supposons que la chaleur n'a pas eu le temps de se propager profondément dans l'échantillon pendant la durée de l'impulsion. On pourra alors le considérer comme ayant une épaisseur infinie. On trouve ainsi en introduisant la puissance absolue maximale $H[6]$ :

$$
T_{\mathrm{f}}=\frac{4 H \tau}{3 D C(2 \pi \alpha \tau)^{1 / 2}}
$$

l'énergie de l'impulsion étant

$$
Q=\frac{1}{2} \beta H \tau,
$$

où $\beta$ est un facteur correctif voisin de 1 tenant compte de l'écart de la courbe de la figure 4 par rapport à un triangle. On peut exprimer $T_{\mathrm{f}}$ en fonction de $Q$, soit

$$
T_{\mathrm{f}}=\frac{8 Q}{3 \beta D C(2 \pi \alpha \tau)^{1 / 2}} .
$$

On a aussi, d'après (1),

$$
T_{\mathrm{f}}=\frac{8}{3 \beta(2 \pi \tau)^{1 / 2}} \cdot \frac{L T_{\mathrm{m}}}{\alpha^{1 / 2}} .
$$

4. Technique expérimentale. - 4.1 ENVIRONNEMENT DES ÉCHANTILLONS. - La validité de la théorie exige en premier lieu un très bon isolement thermique

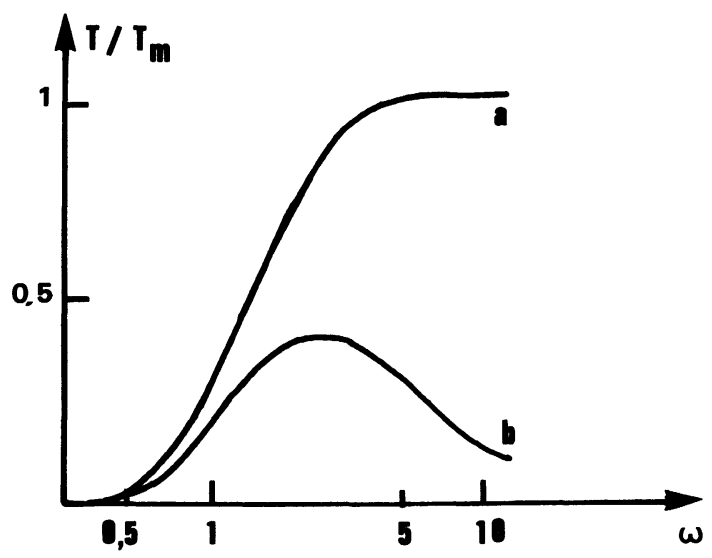

Fig. 6. - Evolution de la température sans pertes (courbe $a$ ) et avec pertes (courbe $b$ ).

[Variation of the temperature without dissipation (curve $a$ ) and with dissipation (curve, $b$ ).] de l'échantillon. Celui-ci se présente sous la forme d'une pastille circulaire de diamètre de $10 \mathrm{~mm}$ et d'épaisseur variable entre 0,2 et $2 \mathrm{~mm}$; elle repose en trois points de sa circonférence sur un support en vitrocéramique MACOR qui assure en même temps l'isolement électrique. Les mesures sont faites sous un vide meilleur que $10^{-3}$ torr grâce à une pompe à zéolithe non polluante, afin d'éliminer les pertes par convection. Les courbes $a$ et $b$ de la figure 6 représentent les variations de $T$ obtenues respectivement en prenant les précautions précédentes et sans limiter les pertes. On voit aisément si l'on a réalisé un isolement suffisant.

Un four électrique entoure l'échantillon; celui-ci peut aussi être refroidi par l'intermédiaire d'une carotte en cuivre plongeant dans l'air liquide. On fait ainsi varier la température d'équilibre. Dans le cas du refroidissement on doit introduire un gaz lorsqu'on fait varier la température de l'échantillon, le vide étant rétabli pour faire la mesure.

Le schéma général de la figure 7 représente l'installation, destinée à fonctionner entre $-170^{\circ} \mathrm{C}$ et $+250^{\circ} \mathrm{C}:$ a est un réflecteur, b est le flash, $\mathrm{c}$ un phototransistor permettant de suivre l'émission de lumière, d est une électrode en cuivre, e l'isolant qui supporte l'échantillon $f$. $g$ est le thermocouple, $h$ le four, $i$ la canalisation de pompage, $\mathrm{j}$ la carotte de cuivre permettant le refroidissement.

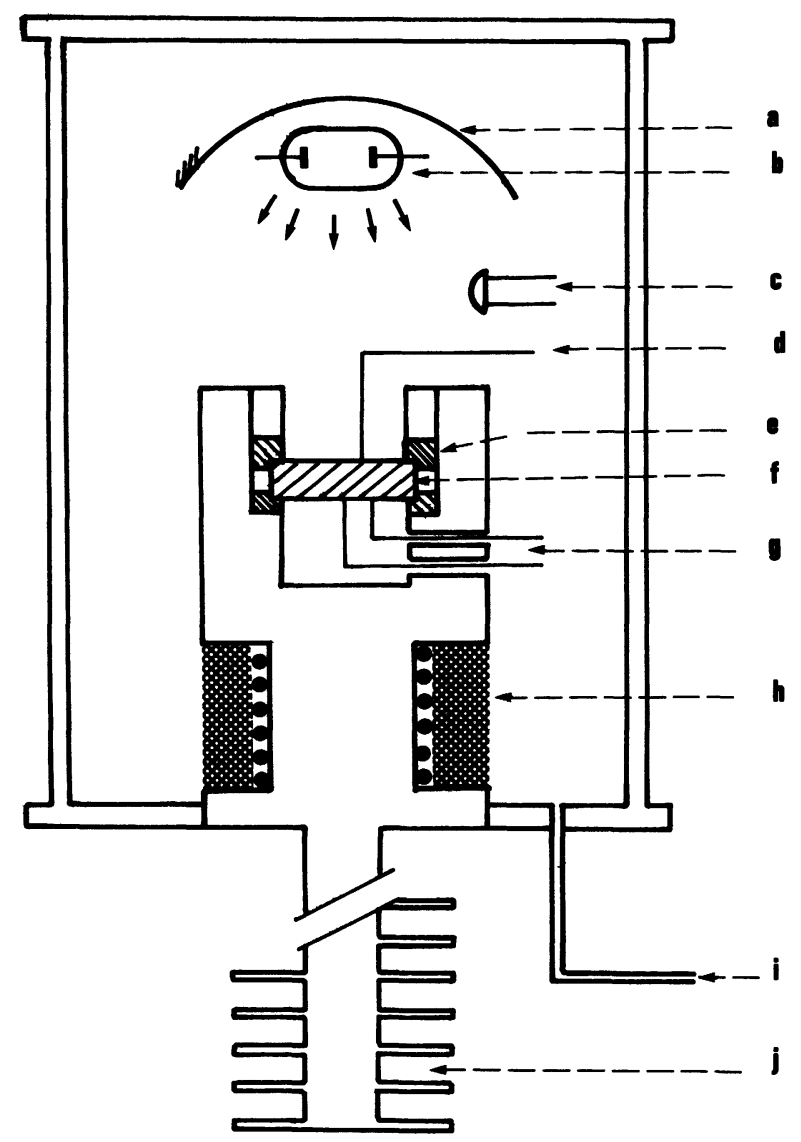

Fig. 7. - Dispositif expérimental.

[Experimental arrangement.] 
4.2 Préparation des ÉChantillons. - Les faces des échantillons non métalliques sont métallisées sous vide avec de l'or afin d'améliorer la répartition du potentiel électrique en surface et d'assurer un contact électrique correct pour le thermocouple. La surface qui reçoit la lumière est en outre noircie au noir de fumée sous l'épaisseur qui permet l'absorption totale de la lumière; un cache évite toute absorption par les faces latérales.

Le thermocouple est du type cuivre-constantan, de diamètre $10 \mu \mathrm{m}$ pour minimiser les pertes par conduction. La jonction est assurée par la couche d'or, les fils du thermocouple faisant contact par pression; cette disposition permet d'obtenir effectivement la température de surface.

Sur la face avant, un fil de cuivre venant en contact par pression permet la mesure de la f.é.m. de Seebeck échantillon/cuivre. Le P.T.E. absolu du cuivre étant connu, on peut déduire de la f.é.m. mesurée le P.T.E. absolu de l'échantillon; pour des semi-conducteurs, on peut négliger le P.T.E. du cuivre.

Le schéma de la figure 8 montre l'échantillon, avec les électrodes de cuivre $a$, l'électrode de constantan $b$, la couche de noir de fumée $\mathrm{c}$, les couches d'or $\mathrm{d}$, le cache e.

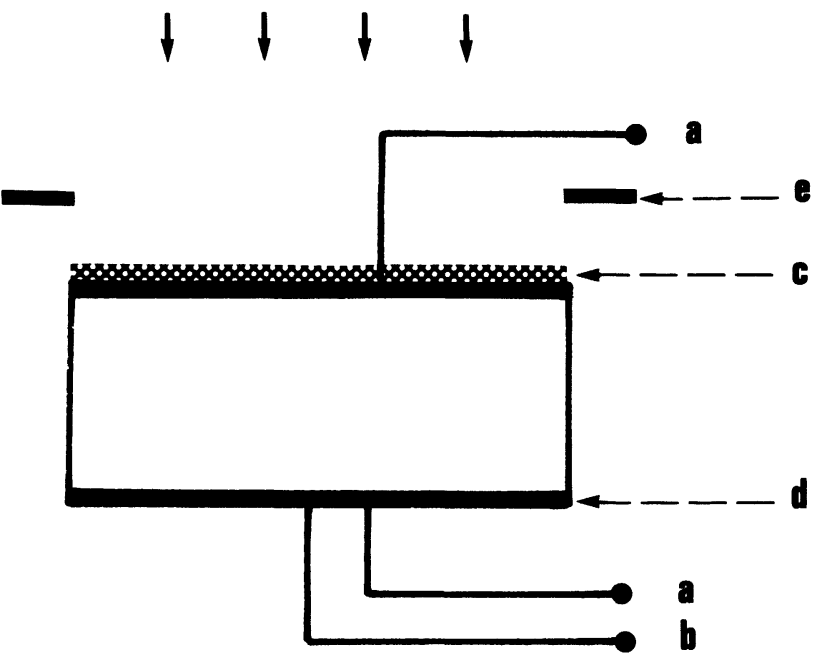

Fig. 8. - L'échantillon avec ses revêtements et ses électrodes.

[The sample with surface layers and electrodes.]

5. Mesures. - Il est nécessaire de déterminer :

- l'énergie fournie par le flash,

- la température initiale $T_{0}$ de l'échantillon,

- la variation $T$ de la température de la face arrière de l'échantillon au cours du temps,

- la variation $\Delta E$ de la f.é.m. de Seebeck aux bornes de l'échantillon en fonction du temps.

5.1 MeSURE DE L'ÉNERGIE. - Dans une première étape nous utilisons comme source lumineuse un flash à commande électronique, dont la puissance est modulable par changement de la valeur de la capacité de stockage.
L'énergie délivrée par le flash peut varier d'un cas à l'autre; il est donc nécessaire de la mesurer. Cette mesure est effectuée par l'intermédiaire du phototransistor, placé, de même que l'échantillon, à une distance invariable du flash. Le signal électrique correspondant à l'éclair lumineux est alors intégré ; la tension continue correspondante est proportionnelle à l'énergie reçue par l'échantillon. Le système de mesure est étalonné à partir d'un échantillon de plomb de caractéristiques connues, pour différentes énergies.

5.2 iMesure De La TeMpérature. - La température moyenne $T_{0}$ est mesurée par l'intermédiaire du thermocouple placé sur la face arrière de l'échantillon.

En ce qui concerne la mesure de $T$, il faut noter que son maximum $T_{\mathrm{m}}$ sera de quelques degrés, donc très inférieur à la variation possible de $T_{0}$ qui a été prévue. Un même appareil de mesure ne peut donc représenter directement à la fois $T_{0}$ et $T$. Pour obtenir $T$ et $T_{0}$ avec des nombres de chiffres significatifs comparables, nous devons amplifier $T$. Or le thermocouple donne $T_{0}+T$. Il faut retrancher $T_{0}$. Pour cela cette quantité $T_{0}$ doit en principe être mise en mémoire, afin d'être conservée pour pouvoir être retranchée. Mais $T_{0}$ étant beaucoup plus grand que $T$, toute perte provenant de la mémoire analogique qui contient $T_{0}$ conduit à une erreur importante. Nous avons éliminé cette difficulté en utilisant la tension de référence $V_{\mathrm{R}}$ du système de régulation de la température $T_{0}$. Si $V_{\mathrm{T}}$ est la tension fournie par le thermocouple, on dispose de la différence $S=V_{\mathrm{R}}-V_{\mathrm{T}}$. A l'instant qui précède un flash lumineux, $S$ prend la valeur $S_{0}$; c'est une quantité relativement faible que l'on mettra dans la mémoire analogique. Ensuite cette tension devient $S_{1}$. La différence $S_{1}-S_{0}$ est la tension qui représente $T$. Ainsi l'erreur due aux pertes de la mémoire sera négligeable.

La figure 9 donne le schéma de principe de la mesure de $T$.

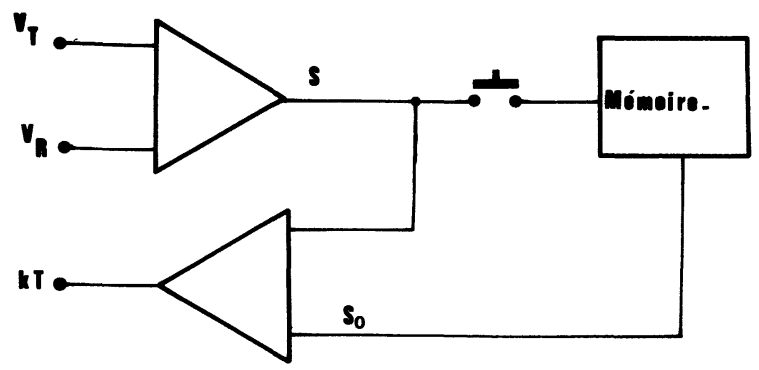

Fig. 9. - Principe de la mesure de la variation $T$ de la température.

[Principle of the measurement of the variation $T$ of the temperature.]

5.3 Mesure De $\Delta E$. - Cette mesure doit être effectuée avec un amplificateur ayant une résistance d'entrée très supérieure à la résistance de l'échantil- 
lon. Nous avons prévu d'opérer sur des échantillons dont la résistance peut atteindre une valeur de $10^{11} \Omega$, avec $t_{1 / 2}>10^{-2}$ set $10^{5}<Q_{\mathrm{V}}<10^{-2} \mathrm{~V}$. Nous avons choisi un amplificateur électromètre à MOS AD $515 \mathrm{~L}$ dont le courant de polarisation est de $10^{-14} \mathrm{~A}$ et le temps de montée de $0,1 \mathrm{~V} / \mu \mathrm{s}$. Le bruit ramené à l'entrée, de l'ordre de $10^{-8} \mathrm{~V} / \mathrm{Hz}^{1 / 2}$, est très compatible avec les niveaux de mesure envisagés. L'amplificateur est monté en adaptateur d'impédance avec gain de 100 ; un étage d'amplification classique peut être monté en cascade. Cet étage est fixé le plus près possible de l'échantillon, afin d'éviter les capacités parasites dues aux fils de liaison. Les valeurs mesurées sont aussi enregistrées.

6. Résultats et conclusion. - La méthode a été essayée comparativement à une méthodè statique dont le principe est illustré par le schéma de la figure 10. L'échantillon du corps à étudier est préparé comme précédemment et posé sur un bloc de cuivre (les deux surfaces en contact ont subi un poli optique), dont la température sera supposée constante et uniforme; c'est aussi la température de la face en contact de l'échantillon. Sa face opposée est chauffée par un faisceau de lumière issu d'une lampe à halogène et focalisé par un système de lentilles. Les tests sont effectés à la température ordinaire; la mesure de la température de la face supérieure est effectuée, lorsqu'un régime stable est établi, à l'aide d'un thermocouple en contact.

La f.é.m. fournie par l'échantillon est alors mesurée à l'aide d'un voltmètre différentiel à haute impédance, entre le bloc de cuivre bien isolé électriquement et une électrode constituée par un fil de cuivre, dont le contact électrique sur la face chaude de l'échantillon est assuré par pression.

Dans ces conditions, la différence entre les résultats obtenus avec cette méthode et la méthode dynamique, avec différents matériaux, n'excédait pas $3 \%$. Ceci confirme la validité de la méthode dynamique.

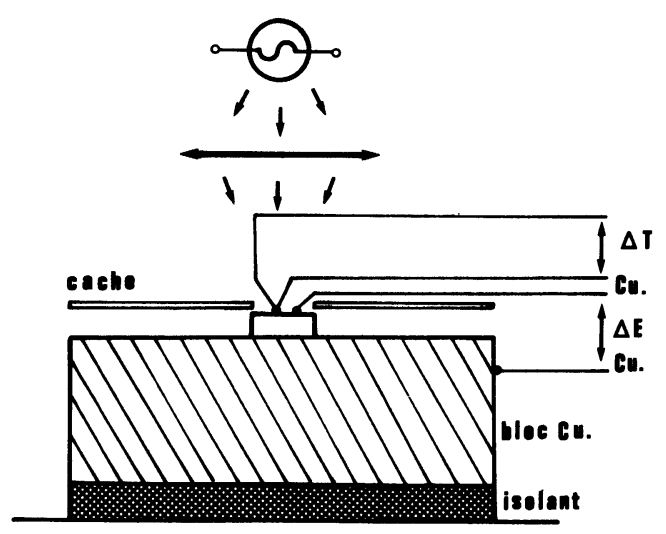

Fig. 10. - Dispositif de mesure statique du pouvoir thermoélectrique.

[Device for static measurement of the thermoelectric power.]
A titre d'exemple, nous donnons sur les figures 11 et 12 les réseaux de courbes correspondant aux mesures les plus significatives effectuées sur des verres semiconducteurs du type chalcogénure quaternaire $\mathrm{Te}_{43} \mathrm{As}_{38} \mathrm{Ge}_{14} \mathrm{~S}_{5}$ contenant différents pourcentages d'or. Ces résultats, obtenus dans une large gamme de températures, complètent d'autres mesures [7] de conductibilité électrique et de bruits de conduction. L'ensemble de ces mesures permet une meilleure compréhension du mécanisme de transport électrique dans ce type de corps.

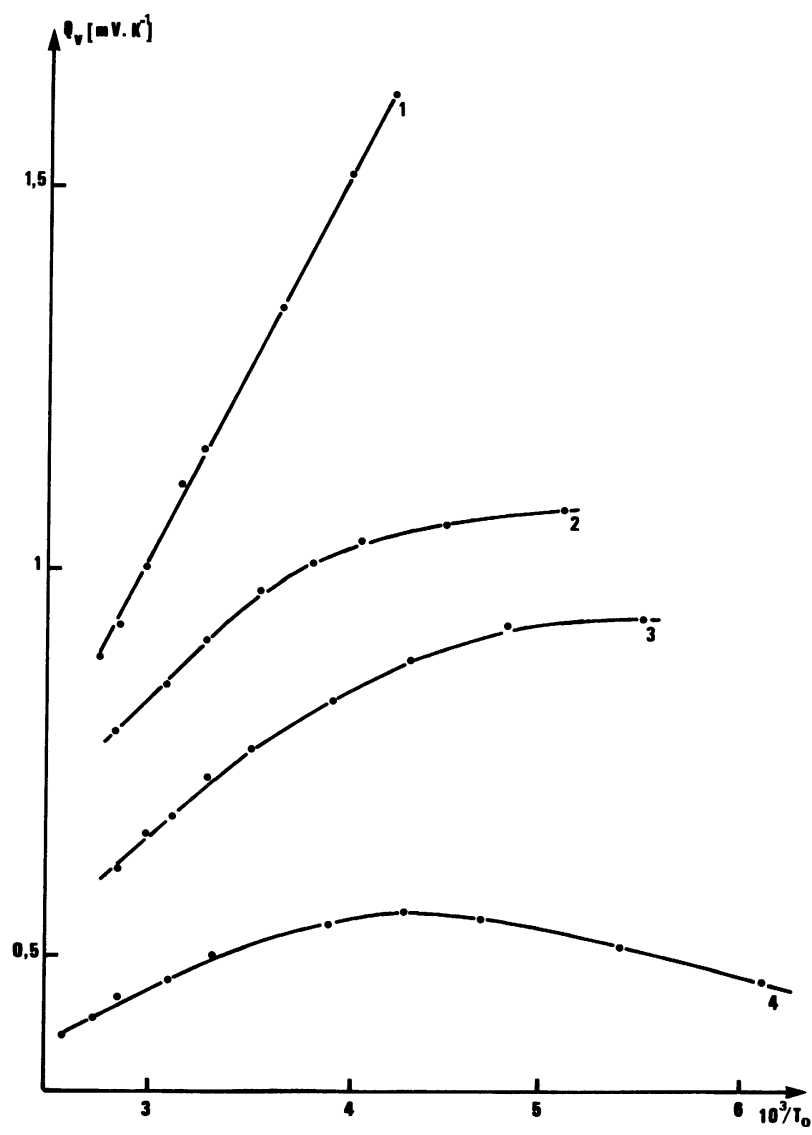

Fig. 11. - Résultats de mesures du pouvoir thermoélectrique en fonction de la température $T_{0}$ pour un chalcogénure dopé. Les courbes 1 à 4 correspondent respectivement à $0 \%, 3 \%, 6 \%$ et $9 \%$ d'or en poids.

[Results of measurements of the thermoelectric power vs. temperature $T_{0}$ for a doped chalcogenide. Curves 1 to 4 correspond respectively to $0 \%, 3 \%, 6 \%$ and $9 \%$ of gold in weight.]

Les courbes de la figure 12 concernent la diffusivité thermique. Elles confirment la structure désordonnée du corps; la longueur de diffusion des phonons, déterminée d'après les courbes, est de l'ordre de $8 \AA$. Sa température a peu d'influence : il en est de même pour la présence d'or, qui ne conduit donc pas à un réarrangement.

D'après le signe du P.T.E., on conclut à une conduction de type $\mathbf{P}$ dans le cas du corps pur; les énergies 


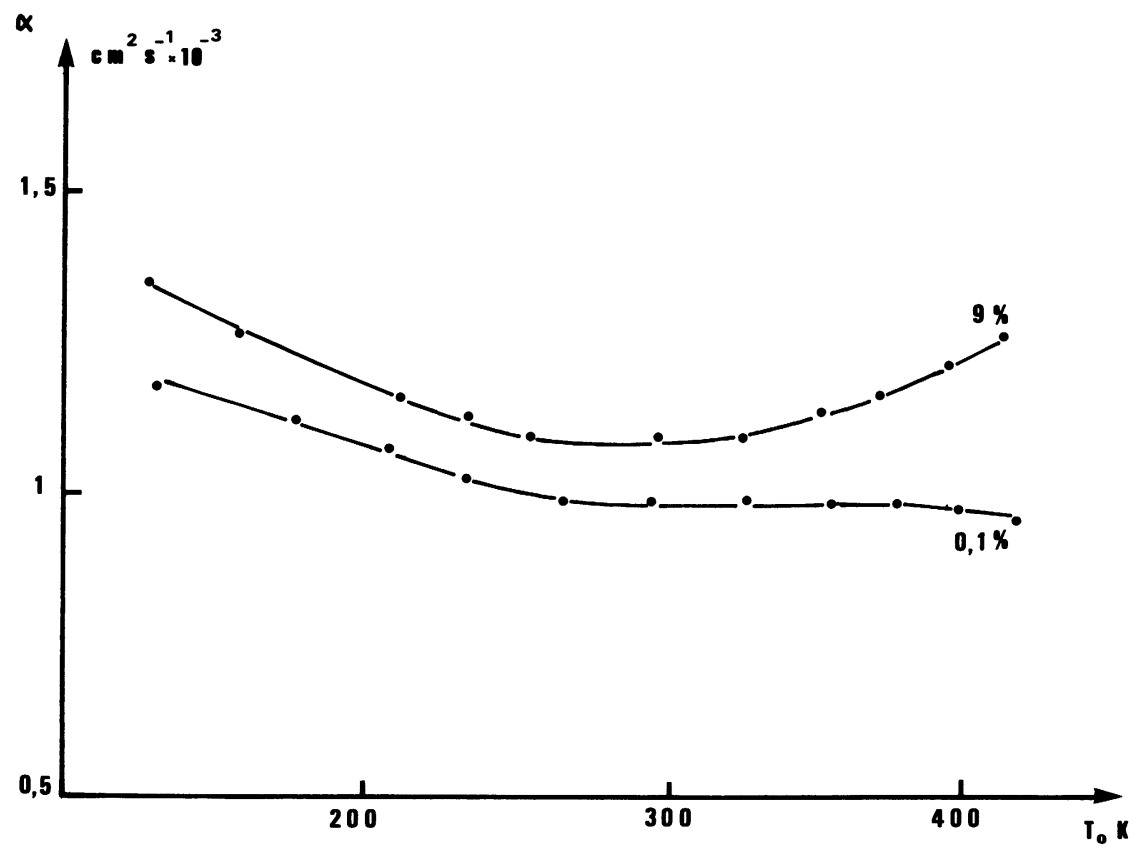

Fig. 12. - Résultats des mesures du coefficient de diffusivité thermique en fonction de la température $T_{0}$. [Results of measurements of the thermal diffusivity coefficient $v s$. temperature $T_{0}$.]

d'activation du P.T.E. et de la conduction électrique [7] sont les mêmes; la conduction est donc due aux porteurs situés dans les états étendus. La détermination du coefficient de température de l'énergie d'activation conduit à la valeur $4 \times 10^{-4}$, d'après la courbe donnant le P.T.E. en fonction de T [8]; on en conclut que le niveau de Fermi n'est pas fixé par des états localisés. Le corps peut alors être représenté par un modèle de bande simple.

Dans le cas d'un dopage à l'or les résultats obtenus nous amènent à un modèle plus complexe. Des états localisés viennent fixer le niveau de Fermi et un mécanisme de transport à deux types de porteurs peut être envisagé (dans le cas de $9 \%$ et de $6 \%$ d'or, on a pu observer un changement du signe du P.T.E.) A haute température la conduction électrique est alors due principalement aux porteurs des états étendus tandis qu'à basse température ce sont surtout ceux des états localisés de la bande interdite qui interviendront [7].

Ainsi en étendant la méthode impulsionnelle de mesure de la diffusivité thermique à la mesure du pouvoir thermoélectrique, nous avons mis au point une technique valable qui permet principalement d'éviter les inconvénients de la mesure directe du gradient thermique. A cet avantage s'ajoute celui de mesures faites en régime dynamique. La précision qui a été trouvée est suffisante dans la plupart des cas.

\section{Bibliographie}

[1] Rowe, V. A. et Schroder, P. A., J. Phys. Chem. Sol. 31 (1970) 1.

[2] Caskey, G. R., Sellmeyer, D. J. et Rubin, L. G., Rev. Scien. Instrum. 40 (1969) 1280.

[3] Conan, A. Goureaux, G. et Zoaeter, M., Revue Phys. Appl. 6 (1971) 8.

[4] Parker, W. J., Jenkins, R. J., Butler, C. P. and Аввотт, G. L., J. Appl. Phys. 32 (1961) 1679.

[5] Heckman, R. C., J. Appl. Phys. 44 (1973) 1455.
[6] Carslaw, H. S. and Jaeger, J. C., Conduction of Heat in solids (Oxford University Press, New York) 1959, 2nd ed., p. 76.

[7] Boukis, D., Thèse de Doctorat de Spécialité, Université Claude Bernard, Lyon, 1979.

[8] MotT, N. F. and Davis, E. A., Electronic processes in non-crystalline solids. The international series of monographs on Physics. 